\title{
Aerobic Exercise Alleviates Inflammation, Oxidative Stress, and Apoptosis in Mice with Chronic Obstructive Pulmonary Disease
}

\author{
Xishuai Wang $\mathbb{1}^{1,2}$ \\ Zhiqing Wang' \\ Donghui Tang'
}

'Department of College of P.E and Sport, Beijing Normal University, Beijing, People's Republic of China; ${ }^{2}$ Department of Animal Genetic Resources, Institute of Animal Science, Chinese Academy of Agricultural Sciences, Beijing, People's Republic of China
Correspondence: Donghui Tang; Xishuai Wang

Department of College of P.E and Sport, Beijing Normal University, No. 19, Xinjiekouwai St, Haidian District, Beijing, I00875, People's Republic of China Email201831070001@mail.bnu.edu.cn; 630200346@qq.com
Background: Chronic inflammation, oxidative stress, and apoptosis play critical roles in chronic obstructive pulmonary disease (COPD) pathogenesis. Here, we attempted to determine whether aerobic exercise (AE) could improve COPD by counteracting the COPDassociated inflammatory response, oxidative stress, and apoptosis in mice.

Methods: Thirty male ICR mice were assigned into one of three groups: control (Con), COPD, and COPD + AE. COPD was simulated by intratracheal injection of lipopolysaccharide (LPS) for 4 weeks. Low-intensity AE was performed for 4 weeks. Bronchoalveolar lavage fluid (BALF) cell counts and the levels of inflammatory cytokine in BALF and serum were detected. Hematoxylin and eosin (HE), Masson trichrome, and Sirius Red staining as well as terminal deoxynucleotidyl transferase dUTP nick end labeling were performed to identify the degree of pulmonary emphysema, bronchial mucus cell hyperplasia, pulmonary fibrosis, and cell apoptosis. Oxidative stress parameters were measured. Furthermore, gene expression levels for the CXCL1, IL-1 $\beta$, IL-10, IL-17, matrix metalloproteinase (MMP)9, TGF- $\beta$, TNF- $\alpha$, and silent information regulator (sirt)1 were detected in mice lung tissues.

Results: AE improved LPS-induced emphysema, pulmonary fibrosis, bronchial mucus cell hyperplasia, bronchoconstriction, and cell apoptosis. AE prevented an LPS-induced increase in the total cell, neutrophil, and macrophage counts. AE decreased malondialdehyde (MDA) and myeloperoxidase (MPO) levels but increased glutathione (GSH) and superoxide dismutase (SOD) levels. AE decreased BALF levels of IL- $1 \beta$, TNF- $\alpha$, and TGF- $\beta$ but increased BALF IL-10 levels. AE suppressed the gene expression levels of pro-inflammatory factors CXCL1, IL-1 $\beta$, IL-17, and TNF- $\alpha$ and profibrotic factors MMP-9 and TGF- $\beta$ but activated those of anti-inflammatory factor IL-10 and lung-protective factor sirt1.

Conclusion: AE is a potential therapeutic approach for COPD. AE improved emphysema, bronchial mucus cell hyperplasia, and pulmonary fibrosis in mice with COPD by alleviating the inflammatory response, oxidative stress injury, and cell apoptosis as well as activating sirt1.

Keywords: exercise, COPD, pulmonary fibrosis, emphysema, pulmonary inflammation, oxidative stress injury

\section{Introduction}

Chronic obstructive pulmonary disease (COPD), which is characterized by lung emphysema, airway remodeling, and not fully reversible airflow limitation, involves alterations in inflammatory parameters and oxidative status. ${ }^{1,2}$ Although significant progress has been made, morbidity and mortality in COPD patients are 
still very high worldwide. ${ }^{3}$ The pathogenesis of COPD is complex. It is well documented that inflammation and oxidative stress play central roles in the pathogenesis of COPD. ${ }^{4,5}$ Moreover, cell apoptosis also contributes to COPD. ${ }^{6}$ We attempted to identify the physiological functions of these factors in response to regular aerobic exercise (AE) in mice with COPD.

COPD is characterized by a chronic inflammatory response involving inflammatory mediators, including interleukin (IL)-1 $\beta$ and $1 \mathrm{~L}-10$, and effector cells, in which neutrophils play an integral role. ${ }^{7,24}$ Deleterious accumulation of neutrophils is a critical mechanism underlying COPD in part because neutrophils release multiple factors, including transforming growth factor beta (TGF- $\beta$ ) and matrix metalloproteinase 9 (MMP-9), which are important in the pathogenesis of COPD. ${ }^{8,9}$ Previous studies have demonstrated that exercise improved immune functions by altering the number of immune cells, including neutrophils. ${ }^{10}$ Inflammatory mediators such as IL-1 $\beta$ and 1L-10, and effector cells, including neutrophils, have been established as crucial mechanisms of COPD, but their physiological functions in response to regular $\mathrm{AE}$ in mice with COPD remain unclear.

Pulmonary fibrosis, which is characterized by progressive dyspnea and hypoxemia; is a serious condition with few available treatments. ${ }^{11}$ Neutrophils release multiple factors, including TGF- $\beta$ and MMP-9, which are essential in the pathogenesis of pulmonary fibrosis. ${ }^{8,9}$ MMP-9 is associated with emphysema, pulmonary inflammation, and lung remodeling in patients with COPD. ${ }^{10}$ Profibrosis factors, such as IL-17, MMP-9, and TGF- $\beta$, also contribute to the pathogenesis of pulmonary fibrosis; ${ }^{12-14}$ however, their physiological functions in response to regular AE in mice with COPD remain unclear.

Pulmonary fibrosis, which is characterized by progressive dyspnea and hypoxemia; leads to expiratory dyspnea; and is associated with an unacceptably high incidence, is a seriously endangered condition with few available treatments. ${ }^{11}$ Neutrophils release multiple factors, including TGF- $\beta$ and MMP-9, which play prominent roles in the pathogenesis of pulmonary fibrosis. ${ }^{8,9}$ MMP-9 is associated with emphysema, pulmonary inflammation, and lung remodeling in patients with COPD. ${ }^{10}$ TGF- $\beta$ plays vital roles in the pathogenesis of pulmonary fibrosis. ${ }^{11}$ Pro-fibrosis factors, such as IL-17, MMP-9, and TGF- $\beta$ play prominent roles in the pathogenesis of pulmonary fibrosis. $^{12-14}$ However, the physiological role of pro- fibrosis factors in response to regular $\mathrm{AE}$ in mice with COPD remains unclear.

Silent information regulator (sirt1) plays a vital role in the development of COPD. ${ }^{15}$ Compared to the normal population, the levels of sirt1 in COPD patients were significantly reduced. ${ }^{16-18}$ Activation of sirt1 by agents, such as resveratrol, protects against COPD because sirt1 modulates the inflammatory/anti-inflammatory and oxidation/antioxidation balances. ${ }^{19}$ Previous studies have found that exercise can activate sirt1 in muscle tissue, increasing the level of cellular $\mathrm{NAD}^{+}$and the NAD/NADH ratio. ${ }^{20}$ In the present study, we determined whether AE could increase sirtl and modulate the inflammatory/antiinflammatory and oxidation/antioxidation balances in COPD mice.

\section{Materials and Methods}

\section{Animals and Experimental Design}

All protocols used in this study were approved by the Animal Experimental Welfare of the Institute of Animal Science, Chinese Academy of Agricultural Sciences (Beijing, China). All experiments were performed in accordance with the Animal Experimental Welfare of the Institute of Animal Science, Chinese Academy of Agricultural Sciences, and the Guide for the Care and Use of Laboratory Animals published by the US National Institutes of Health. The authors complied with the ARRIVE guidelines and every effort was made to minimize suffering. Thirty male ICR mice (6 weeks old, 18-21 g) were provided by Beijing HFK Biotechnology Co., Ltd. (Beijing, China). The conditions of the husbandry room were temperature at $22 \pm 5{ }^{\circ} \mathrm{C}$, relative humidity at $50 \% \pm 10 \%$, and the light cycle was $12 \mathrm{~h} /$ day. The body weight and health status of the mice were recorded. The mice were anesthetized via intraperitoneal injection of pentobarbital $(50 \mathrm{mg} / \mathrm{kg})$.

The COPD model was produced by intratracheal injection of $0.2 \mathrm{mg} / \mathrm{kg}$ LPS (L2880) twice a week for 4 weeks as previously described. $^{21-25}$ The mice were divided into three groups with eight individuals each: (1) control (Con), in which mice received the same volume of saline via intratracheal injection; (2) COPD, in which mice received $0.2 \mathrm{mg} / \mathrm{kg}$ LPS via intratracheal injection; or (3) COPD + AE, in which mice received $0.2 \mathrm{mg} / \mathrm{kg}$ LPS via intratracheal injection and were subjected to AE for 4 weeks, simultaneously. LPS was dissolved in saline solution. Saline or LPS was injected twice a week (days 1, 4, 8, 11, 15, 18, 22, and 25); thus, the mice 
received a total of eight injections of LPS or saline. All mice were anesthetized via intraperitoneal injection of pentobarbital $(50 \mathrm{mg} / \mathrm{kg})$. All mice were euthanized on day 28 .

\section{Exercise Protocol}

The mice were trained using a six-lane treadmill. Adaptive training of the mice was performed for 3 days $(20 \mathrm{~min}$, $25 \%$ inclination, $3.3 \mathrm{~m} / \mathrm{min}$ ). After the adaptive training, the maximal exercise capacity test of each mouse was performed. The maximal aerobic capacity was determined based on the maximal speed. The mean maximal speed reached by each animal was $23.6 \mathrm{~m} / \mathrm{s}$. Hence, the maximal aerobic capacity (100\%) was $23.6 \mathrm{~m} / \mathrm{s}$. Mice from AE and COPD + AE groups were trained in low-intensity exercise ( $50 \%$ maximal speed, $11.8 \mathrm{~m} / \mathrm{s}$ ). The exercise intervention lasted for 4 weeks, 5 days/week, 1 h/day.

\section{Collection of Tissue Samples}

Blood samples were collected and placed in a blood collection vessel containing an anticoagulant, followed by centrifugation at $3000 \mathrm{rpm}$ for $15 \mathrm{~min}$. The serum was transferred to Ep tubes and immediately stored at $-20^{\circ} \mathrm{C}$. We collected bronchoalveolar lavage fluid (BALF) using $2 \mathrm{~mL}$ of ice-cold $0.9 \% \mathrm{NaCl}$. The whole lung was flushed four times, and the returned fluid was collected. The supernatant was stored at $-80{ }^{\circ} \mathrm{C}$ until subsequent analysis. After sampling, the lung tissue samples were immediately placed in an incubator filled with liquid nitrogen. The tissue was then transferred to a liquid nitrogen tank for long-term preservation. For histological analysis, lung tissue samples were fixed in 4\% paraformaldehyde (PFA).

\section{Histopathology}

For histological analysis, 4\% PFA-fixed lung tissue samples were embedded in paraffin. Using the paraffin section method, 5- $\mu \mathrm{m}$-thick sections were stained with HE, Masson, Sirius Red, and TUNEL. Photographs taken using an inverted microscope (Olympus Corp., Japan) were used to identify the degree of pulmonary emphysema, pulmonary fibrosis, and cell apoptosis.

\section{Detection of Pulmonary and Systemic Inflammation}

The levels of the pro-inflammatory factors CXCL1, IL-17, TGF- $\beta$, IL- $1 \beta$, and TNF- $\alpha$ as well as that of the antiinflammatory factor IL-10 in the BALF were detected using mouse enzyme-linked immunosorbent assay
(ELISA) kits (NeoBioscience Technology Company, Shenzhen, China). Similarly, the levels of the TNF- $\alpha$ and IL-10 in serum were detected using mouse ELISA kits (NeoBioscience Technology Company, Shenzhen, China).

\section{Determination of the Gene Expression Levels in Lung Tissue}

RNA from lung tissues was extracted using TRIzol (Promega, Madison, WI), their concentration was measured to detect the quality of total RNA, and cDNA was synthesized using an Applied Biosystems 2720 Thermal Cycler (Applied Biosystems, Singapore). GAPDH was selected as the internal reference gene, and the $2^{-\Delta \Delta C T}$ method was used for quantitative analysis. All primers were designed and synthesized by the Shanghai Sangon Biotech Company (Shanghai, China). Table 1 lists the murine PCR primer sequences.

\section{Statistical Analysis}

SPSS 21.0 software was used for data processing. The experimental results are indicated by the mean \pm standard deviation $(\mathrm{x} \pm \mathrm{SD})$. Statistical differences among groups

Table I PCR Primer Sequence Information of Mouse

\begin{tabular}{|c|c|}
\hline Gene Name & Primer Sequence \\
\hline \multirow[t]{2}{*}{ IL-I $\beta$} & F: 5' GCTCATTGCTGGGTACTTACAA 3' \\
\hline & R: 5'CCAGACTTGGCACAAGACAGG 3' \\
\hline \multirow[t]{2}{*}{ IL-I0 } & F: 5' GCTCTTACTGACTGGCATGAG 3' \\
\hline & R: 5' CGCAGCTCTAGGAGCATGTG 3' \\
\hline \multirow[t]{2}{*}{ IL-17 } & F: 5' CCAAGAGGTGAGTGCTTCCC 3' \\
\hline & R: 5' CTGTTGTTCAGACTCTCTCCCT 3' \\
\hline \multirow[t]{2}{*}{ TNF- $\alpha$} & F: 5' ATGTCTCAGCCTCTTCTCATTC 3' \\
\hline & R: 5' GCTTGTCACTCGAATTTTGAGA 3' \\
\hline \multirow[t]{2}{*}{ CXCLI } & F: 5' CTGGGATTCACCTCAAGAACATC 3' \\
\hline & R: 5'CAGGGTCAAGGCAAGCCTC 3' \\
\hline \multirow[t]{2}{*}{ Sirtl } & F: 5' GCTGACGACTTCGACGACG 3' \\
\hline & R: 5' TCGGTCAACAGGAGGTTGTCT 3' \\
\hline \multirow[t]{2}{*}{ MMP-9 } & F: 5' TCTTGGAGTAAGTCGAGAAGTGT 3' \\
\hline & R: 5' GTTGAAACTGAGCGAAAAAGGC 3' \\
\hline \multirow[t]{2}{*}{ GAPDH } & F: 5' AGGTCGGTGTGAACGGATTTG 3' \\
\hline & R: 5' TGTAGACCATGTAGTTGAGGTCA 3' \\
\hline
\end{tabular}

Abbreviation: $\mathrm{PCR}$, polymerase chain reaction. 
were assessed using one-way analysis of variance (ANOVA). The significance level was set at $P<0.05$. GraphPad Prism 6 software was used to draw figures.

\section{Results}

\section{Protective Effects of AE Against Pulmonary Inflammation}

After LPS administration for $6 \mathrm{~h}$, the number of total cells $(P<0.001)$, neutrophils $(P<0.001)$, and macrophages $(P<$ $0.001)$ were significantly increased. AE played an essential role in preventing the increase in the number of total cells $(P=0.0374)$, neutrophils $(P=0.0203)$, and macrophages $(P=0.0236)$ compared to the COPD group. Treatment with LPS and AE did not influence the number of lymphocytes $(P>0.05)$ or eosinophils $(P>0.05)$ in the BALF (Table 2$)$.

LPS administration markedly upregulated BALF levels of CXCL1 $(P<0.001)$, IL-1 $\beta(P<0.001)$, IL-10 $(P<$ $0.001)$, IL-17 $(P<0.001)$, TNF- $\alpha(P<0.001)$ and TGF- $\beta$ $(P<0.001)$ compared to the Con group. AE upregulated BALF levels of IL-10 $(P=0.0014)$ and CXCL1 $(P=$ $0.0036)$; downregulated BALF levels of TGF- $\beta(P=$ $0.0285)$, IL-1 $\beta(P=0.0378)$, and TNF- $\alpha(P=0.0284)$; and did not influence BALF levels of IL-17 $(P=0.96)$ compared to the COPD group. These results demonstrate that the 4-week AE regimen relieved pulmonary inflammation in mice with COPD (Table 3).

\section{Protective Effects of AE Against Systemic Inflammation}

The serum levels of IL-10 $(P<0.001)$ and TNF- $\alpha(P=$ 0.012 ) were significantly increased after compared to the Con group. AE upregulated serum levels of IL-10 ( $P=$ $0.0062)$ and downregulated serum levels of TNF- $\alpha(P=$ 0.0249) in mice with COPD (Table 3). These results demonstrate that the 4-week AE relieved systemic inflammation in mice with COPD.

\section{Protective Effects of AE Against Lung Emphysema and Airway Remodeling}

HE staining revealed the degree of emphysema. Compared to the Con group (Figure 1A), LPS administration increased the mean linear intercept (MLI; Figure 1B). 4 weeks of AE significantly decreased the MLI (Figure 1C) compared to the COPD group. Similarly, the MLI

Table 2 Total and Differential Cell Counts in BALF (Cells/ml)

\begin{tabular}{|l|c|c|c|c|}
\hline & Con & COPD & COPD + AE & P \\
\hline Total cells & $1.29 \pm 0.86$ & $9.98 \pm 2.14^{\#}$ & $8.08 \pm 0.94^{*}$ & $<0.001$ \\
Neutrophils & $0.04 \pm 0.04$ & $7.55 \pm 1.23^{\#}$ & $5.84 \pm 1.38^{*}$ & $<.001$ \\
Lymphocytes & $0.03 \pm 0.05$ & $0.05 \pm 0.14$ & $0.05 \pm 0.16$ & 0.88 \\
Macrophages & $1.09 \pm 0.48$ & $2.12 \pm 0.77$ & $1.26 \pm 0.57$ & 0.007 \\
Eosinophils & $0.01 \pm 0.03$ & $0.01 \pm 0.03$ & $0.04 \pm 0.05$ & 0.21 \\
\hline
\end{tabular}

Notes: Compared with the Con group, LPS administration increased the number of total cells, neutrophils, and macrophages. Compared with the LPS group, exercise administration decreased the number of total cells, neutrophils, and macrophages. \#Indicates that the difference in the data is significant compared with the Con group *Indicates that the difference in the data is significant compared with the COPD group. Values are expressed as the means \pm SD.

Table 3 The Levels of Inflammatory Factors in BALF and Serum (pg/ml)

\begin{tabular}{|c|c|c|c|c|}
\hline & Con & COPD & COPD + AE & $\mathbf{P}$ \\
\hline CXCLI BALF & $9.24 \pm 3.65$ & $1106.6 \pm\left. 357.3\right|^{\#}$ & $1648.91 \pm 256.32^{*}$ & $<0.001$ \\
\hline IL-I7 BALF & $8.31 \pm 1.33$ & $254.16 \pm 97.69^{\#}$ & $251.44 \pm 108.92$ & $<0.001$ \\
\hline TGF- $\beta$ BALF & $21.35 \pm 4.86$ & $165.68 \pm 63.75^{\#}$ & $98.45 \pm 44.73^{*}$ & $<0.001$ \\
\hline IL-I0 BALF & $26.9 \pm 20.7$ & $503.2 \pm 95.85^{\#}$ & $834.9 \pm 216.7^{*}$ & $<0.001$ \\
\hline IL-I $\beta$ BALF & $12.65 \pm 2.14$ & $706.9 \pm 141.5^{\#}$ & $563.1 \pm 106.93 *$ & $<0.001$ \\
\hline TNF- $\alpha$ BALF & $|3.3| \pm 5.92$ & $152.3 \pm 28.9^{\#}$ & $124.6 \pm 13.9 *$ & $<0.001$ \\
\hline IL-I0 serum & $13.19 \pm 15.65$ & $506.6 \pm|57.3|^{\#}$ & $748.9 \pm 143.3^{*}$ & $<0.001$ \\
\hline TNF- $\alpha$ serum & $14.24 \pm 8.46$ & $54.45 \pm\left. 16.8\right|^{\#}$ & $31.1 \pm 10.21 *$ & $<0.001$ \\
\hline
\end{tabular}

Notes: The levels of CXCLI, IL-I7, TGF- $\beta$, IL-10, IL-I $\beta$, and TNF- $\alpha$ in BALF and the levels of IL-I0 and TNF- $\alpha$ in serum were detected. ${ }^{\#}$ Indicates that the difference in the data is significant compared with the Con group, *Indicates that the difference in the data is significant compared with the COPD group. Values are expressed as the means \pm SD.

Abbreviations: IL, interleukin; TNF, tumor necrosis factor; CXCL, C-X-C motif chemokine ligand; TGF, transforming growth factor. 

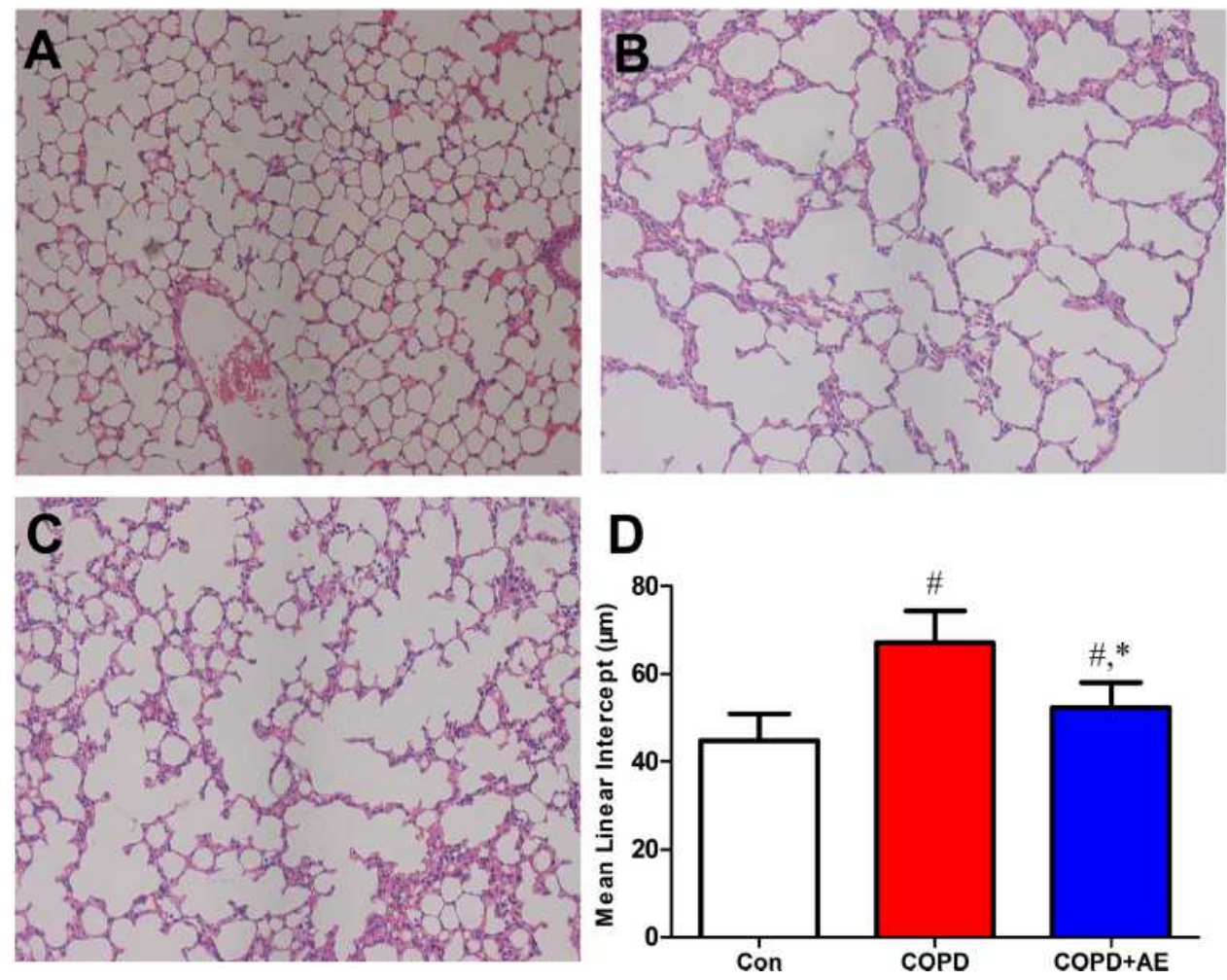

Figure I Detection of emphysema.

Notes: (A) Con group. (B) COPD group. (C) COPD+AE group. Hematoxylin-eosin staining revealed evidence of the degree of emphysema. (D) Detection of MLI. LPS administration increased the MLI, while 4 weeks of exercise intervention significantly decreased the MLI. The difference in MLI was significant between the Con and COPD + AE groups. ${ }^{\#} P<0.05$ compared with the Con group. ${ }^{*} P<0.05$ compared with the COPD group (magnification, $x \mid 00$ ).

Abbreviation: MLI, mean linear intercept.

increased after LPS administration $(P<0.001)$, whereas exercise intervention decreased the MLI $(P=0.0005)$ (Figure 1D). The difference in the MLI between the Con and COPD + AE groups was significant $(P=0.0218)$.

\section{Protective Effects of AE Against Pulmonary Fibrosis}

Masson staining revealed the degree of pulmonary fibrosis. Compared to that in the Con group (Figure 2A), the degree of pulmonary fibrosis in the airway wall increased after LPS administration (Figure 2B). Compared to the COPD group, exercise intervention decreased the degree of pulmonary fibrosis in the airway wall (Figure 2C). Similarly, the fibrotic score increased after LPS administration $(P<$ $0.001)$, whereas exercise intervention decreased the fibrotic score $(P<0.001$; Figure 2D).

Sirius Red staining revealed evidence of collagen fiber deposition. Compared to the Con group (Figure 3A), LPS administration increased collagen fiber deposition in the airway wall (Figure 3B). Compared to the COPD group, 4 weeks of exercise intervention decreased collagen fiber deposition in the airway wall (Figure 3C). Similarly, airway collagen increased after LPS administration $(P<$ $0.001)$, whereas exercise intervention decreased the fibrotic score $(P<0.001$; Figure $3 \mathrm{D})$.

LPS administration increased the levels of pro-fibrotic factor TGF- $\beta$ in the BALF compared to the Con group $(P<$ 0.001; Table 3). Compared to the COPD group, AE decreased the levels of TGF- $\beta(P=0.0285)$ in the BALF. LPS administration increased the gene expression levels of profibrotic factors IL-17 $(P<0.001$; Figure $4 \mathrm{C})$, TGF- $\beta(P<0.001$; Figure 4F), and MMP-9 ( $P<0.001$; Figure 4G) compared to the Con group. 4-week AE regimen decreased gene expression levels of IL-17 $(P<0.001)$, TGF- $\beta(P<0.001)$, and MMP-9 $(P<0.001)$ compared to the COPD group.

\section{Protective Effects of AE Against Cell Apoptosis}

TUNEL staining revealed evidence of cell apoptosis in the lung tissue. In the control group, a small number of cells were apoptotic (Figure 5A), and many apoptotic cells were detected after LPS administration (Figure 5B). AE of 

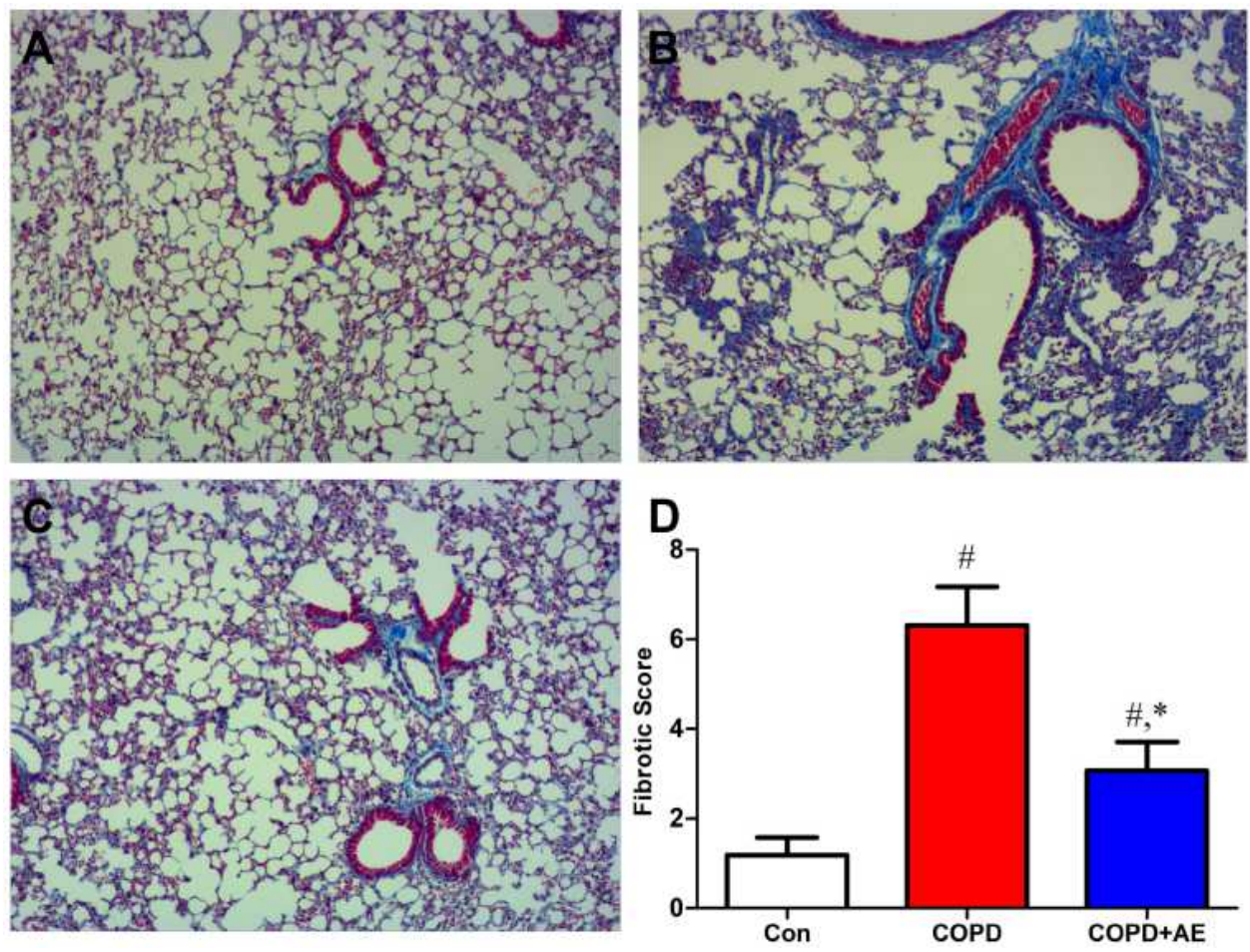

Figure 2 Detection of pulmonary fibrosis.

Notes: (A) Con group. (B) COPD group. (C) COPD+AE group. (D) Detection of the fibrotic score. Mason staining revealed evidence of the degree of pulmonary fibrosis. The fibrotic score increased after LPS administration, while exercise administration decreased fibrotic score. ${ }^{\#} P<0.05$ compared with the Con group. ${ }^{*} P<0.05$ compared with the COPD group (magnification, $\times 200$ ).

COPD mice inhibited the number of apoptotic cells (Figure 5C). Similarly, the apoptosis index increased after LPS administration $(P<0.001)$, while exercise intervention decreased it in mice with COPD $(P<0.001$; Figure 5D).

\section{AE Attenuates Oxidative Stress Injury in Lung Tissue}

The levels of MDA (Figure 6A), MPO (Figure 6B), GSH (Figure 6C), and SOD (Figure 6D) were detected as indicators of oxidative stress injury. After LPS administration, the levels of MDA $(P<0.001)$ and MPO $(P<0.001)$ increased, while the levels of SOD $(P<0.001)$ and GSH $(P<0.001)$ decreased. Compared to the COPD group, 4 weeks of exercise intervention upregulated the levels of SOD $(P<0.001)$ and GSH $(P<0.001)$ but downregulated the levels of MDA $(P<0.001)$ and MPO $(P<0.001)$.

\section{Effects of LPS Injection and AE on Gene Expression Levels}

LPS injection upregulated the gene expression levels of proinflammatory factors IL-1 $\beta(P<0.001$; Figure 4A), IL-17 $(P<$
0.001; Figure 4C), TNF- $\alpha(P<0.001$; Figure 4D), and CXCL1 $(P<0.001$; Figure 4E) as well as those of profibrotic factors TGF- $\beta(P<0.001$; Figure 4F) and MMP-9 $(P<0.001$; Figure $4 \mathrm{G})$ while downregulating the levels of anti-inflammatory factor IL-10 $(P<0.001$; Figure 4B) and lung protective factor sirt1 $(P<0.001$; Figure $4 \mathrm{H})$. AE of COPD mice activated the gene expression levels of IL-10 $(P<0.001)$ and sirt1 $(P<$ $0.001)$ but inhibited gene expression levels of CXCL1 $(P<$ 0.001), IL-1 $\beta(P<0.001)$, IL-17 $(P<0.001)$, MMP-9 $(P<$ $0.001)$, TGF- $\beta(P<0.001)$, and TNF- $\alpha(P<0.001)$.

\section{Detection of Bronchial Mucus Cell Hyperplasia and Bronchoconstriction}

Compared to the Con group (Figure 7A), LPS administration led to severe bronchial mucus cell hyperplasia and bronchoconstriction (Figure 7B). Compared to the COPD group, 4 weeks of exercise intervention improved bronchial mucus cell hyperplasia and bronchoconstriction (Figure 7C).

\section{Discussion}

The present study aimed to investigate whether AE could protect against LPS-induced inflammation, oxidative 

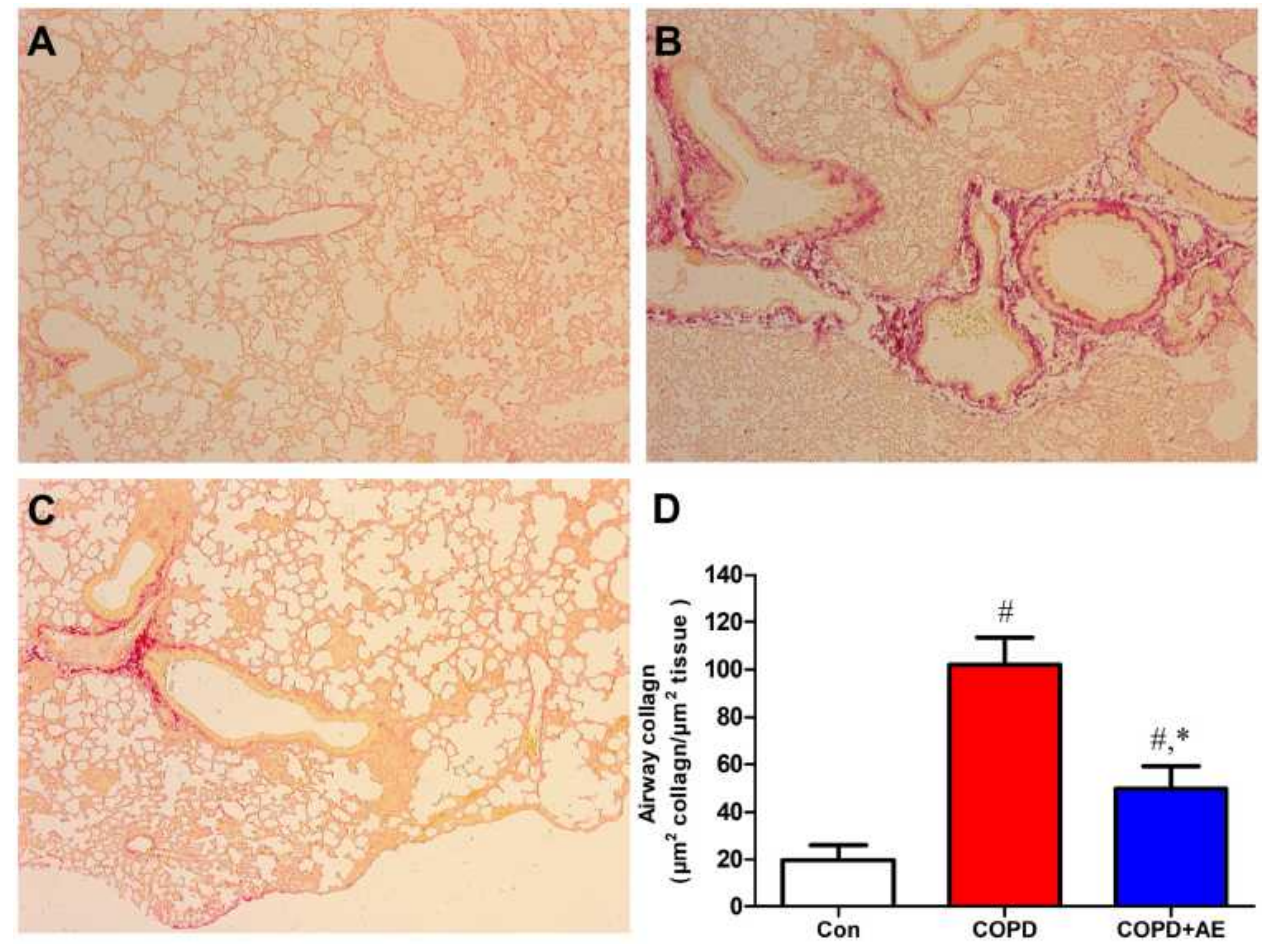

Figure 3 Detection of airway fibrosis.

Notes: (A) Con group. (B) COPD group. (C) COPD+AE group. (D) Detection of collagen fiber deposition in the airway wall. The airway collagen increased after LPS administration, while exercise administration decreased fibrotic score. The results were expressed as $\mu m^{2}$ of collagen fibers per $\mu m^{2}$ of tissue area. ${ }^{\#} P<0.05$ compared with the Con group. $* P<0.05$ compared with the COPD group (magnification, $x 40$ ).

stress, and apoptosis in mice with COPD and explore the related mechanisms.

Previous studies have demonstrated that LPS administration induces an inflammatory response, neutrophil recruitment, emphysema, bronchial mucus cell hyperplasia, and bronchoconstriction, which are the main features of COPD. ${ }^{21}$ The experimental results were consistent with previous conclusions. $^{21-25}$ The results of Masson and Sirius Red staining demonstrated that 4 weeks of $\mathrm{AE}$ prevented the upregulation of the degree of pulmonary fibrosis, suggesting that $\mathrm{AE}$ had an anti-fibrotic effect. We found that LPS injection increased the BALF levels of pro-fibrogenic factors (IL-17 and TGF- $\beta$ ) and increased the gene expression levels of IL-17, MMP-9, and TGF- $\beta$ in the lung tissue. In contrast, $\mathrm{AE}$ inhibited the levels of TGF- $\beta$ in the BALF and decreased the gene expression levels of IL-17, MMP-9, and TGF- $\beta$, suggesting that AE exhibited an anti-fibrogenic effect in mice with COPD.

Previous studies have found that TNF- $\alpha$ is chemotactic for neutrophils, and anti-TNF- $\alpha$ antiserum administration reduces neutrophil influx into bronchoalveolar spaces. ${ }^{21}$ In the present study, we found that AE reduced the levels of TNF- $\alpha$ in serum and the BALF as well as the gene expression levels of TNF- $\alpha$ in the lung tissue. We also found that LPS administration led to a deleterious accumulation of neutrophils in the lung tissue, while 4 weeks of AE inhibited neutrophil infiltration in mice with COPD. These results demonstrate that AE inhibits neutrophil infiltration in part by decreasing TNF- $\alpha$ levels.

Neutrophils release a variety of cytokines, including MMP-9 and TGF- $\beta$, which play prominent roles in the development of COPD. ${ }^{12-14}$ A 4-week AE regimen prevented the upregulation of TGF- $\beta$ and MMP-9 levels in mice with COPD. These results demonstrated that $\mathrm{AE}$ improved COPD in part by decreasing the levels of MMP-9 and TGF- $\beta$. Chronic inflammation characterized by increasing pro-inflammatory factor levels and decreasing anti-inflammatory factor levels is closely related to COPD and that this association is causal. ${ }^{26}$ In the present study, AE decreased the gene expression levels of CXCL1, IL-1 $\beta$, IL-17, and TNF- $\alpha$ while increasing the gene expression levels of IL-10. Similarly, AE decreased BALF levels of IL- $1 \beta$, TNF- $\alpha$, and TGF- $\beta$, while AE increased the IL10 levels. These results demonstrate that regular $\mathrm{AE}$ in COPD mice is essential in modulating the inflammatory/ anti-inflammatory balance. 

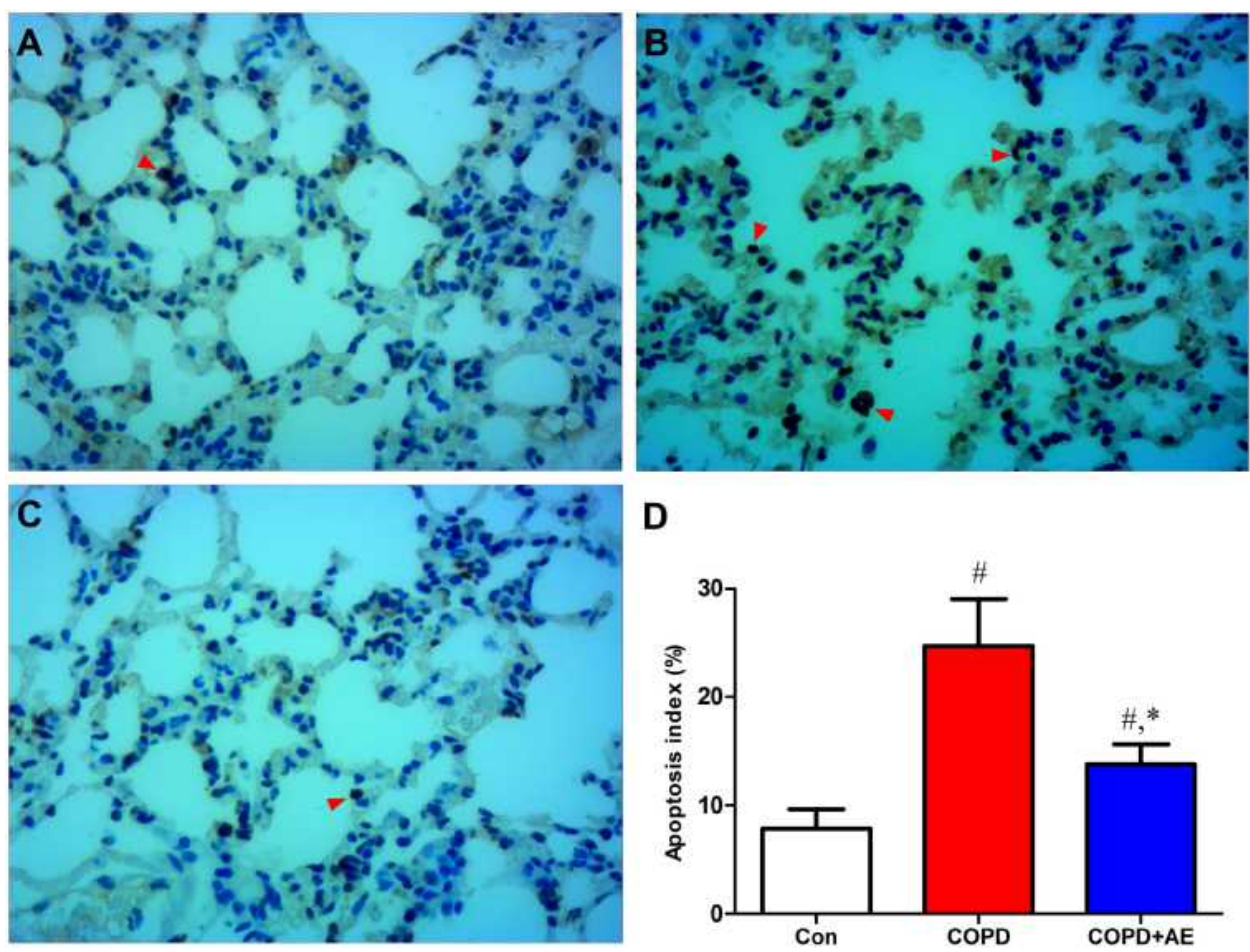

Figure 4 Detection of gene expression levels in lung tissue.

Notes: The gene expression levels of pro-inflammatory factors IL-I $\beta$ (A), IL-I7 (C), TNF- $\alpha$ (D), and CXCLI (E) and profibrotic factors TGF- $\beta$ (F) and MMP-9 (G) and decreased anti-inflammatory factor IL-I0 (B) and lung-protective factor sirt I $(\mathbf{H})$ were detected. ${ }^{*} P<0.05$ compared with the Con group. ${ }^{* P}<0.05$ compared with the COPD group.

Previous studies have found that the oxidant/antioxidant balance plays a prominent role in the pathogenesis of COPD, and exercise improves COPD by exerting protective effects against oxidative stress through the exercise-irisin-nuclear factor erythroid 2-related factor 2 axis in mice. ${ }^{5,27}$ In the present study, we identified the antioxidant effects of exercise. We demonstrated that $\mathrm{AE}$ in COPD mice downregulated the levels of MDA and MPO while upregulating the levels of SOD and GSH. Hence, AE attenuated oxidative stress injury and modulated the oxidative/antioxidative balance in mice with COPD. AE is a preventive tool for COPD, and one of the mechanisms involved in this process is linked to the increase in antioxidant capacity. Moreover, sirt1 has a therapeutic effect in a COPD model, which is related to the inhibition of oxidative stress. ${ }^{18}$ These results demonstrated that $\mathrm{AE}$ attenuated oxidative stress in part by activating sirt1 expression in mice with COPD.

Physical exercise has been frequently used as a therapeutic tool for COPD. One of the mechanisms involved in this process is associated with increases in antioxidant and anti-inflammatory properties. Cell apoptosis is also responsible for the pathogenesis of COPD. However, the effect of $\mathrm{AE}$ on cell apoptosis in mice with
COPD has been overlooked. ${ }^{6}$ In the present study, we found that 4 weeks of $\mathrm{AE}$ inhibited apoptosis in mice with COPD. Hence, AE improved COPD in part by inhibiting cell apoptosis. To the best of our knowledge, this is the first study to demonstrate that $\mathrm{AE}$ exerts a significant protective effect against apoptosis in mice with COPD.

Sirt1 is an important lung-protective factor, but its physiological roles in the response to AE in COPD mice are unknown. We identified that LPS administration inhibited the gene expression levels of sirt1, while a 4-week AE regimen activated these levels. By activating sirt1 expression, AE improved COPD.

\section{Limitations}

AE reduced apoptosis in mice with COPD. However, the underlying mechanism by which exercise reduces apoptosis remains unclear. Even though AE could improve exercise capacity, we did not account for the effects of exercise on the mice. Furthermore, we also did not account for the effects of exercise on the complications of COPD, such as cardiovascular comorbidities, pulmonary arterial hypertension, and muscle wasting. 
A
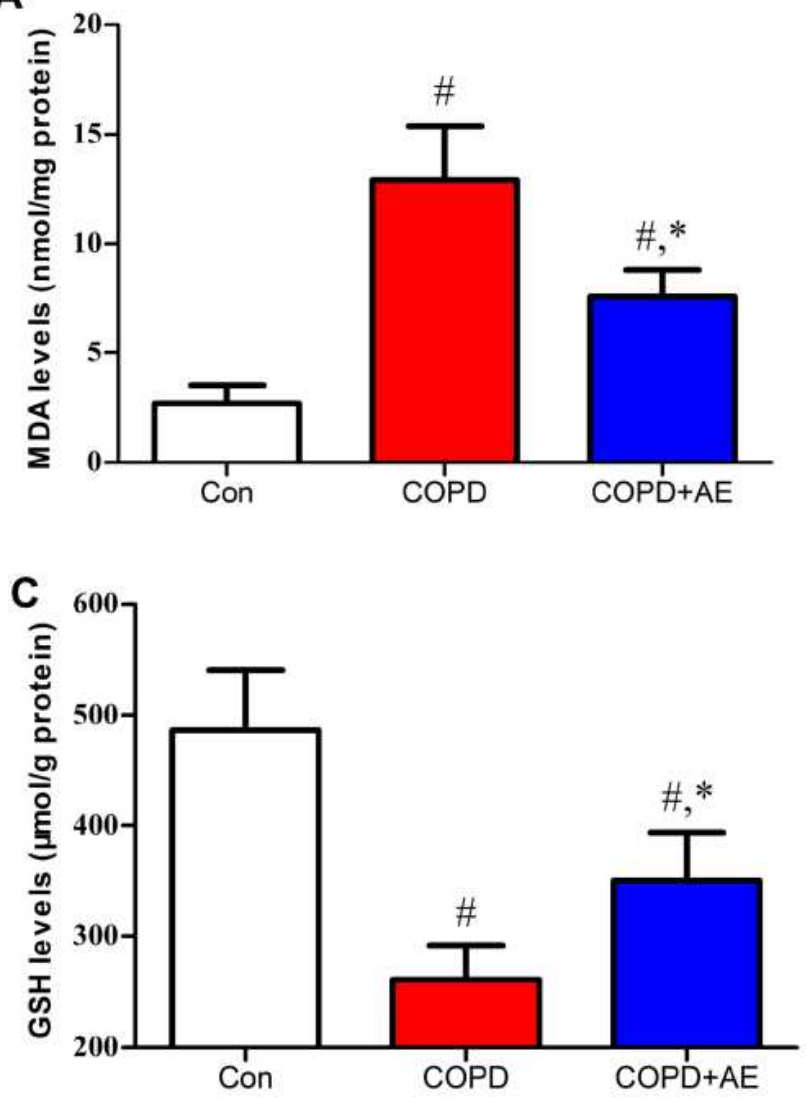

B
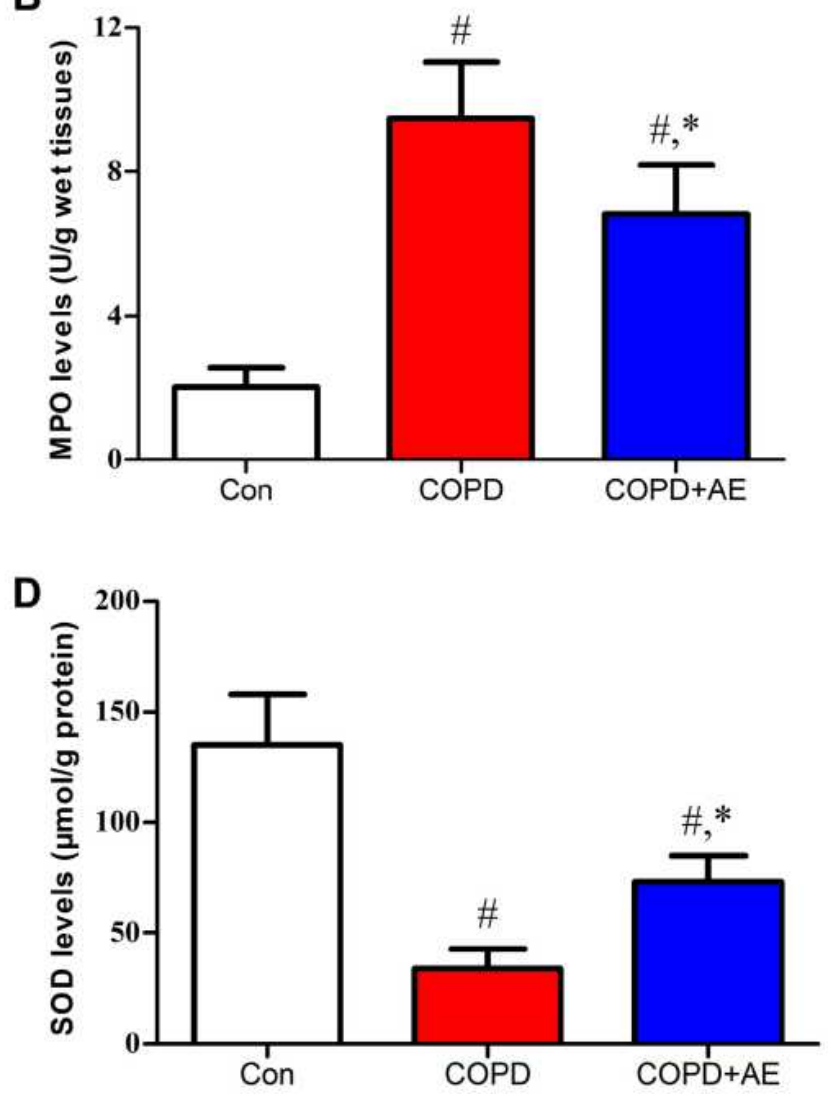

Figure 5 Detection of cell apoptosis.

Notes: (A) Con group. (B) COPD group. (C) COPD+AE group. (D) Detection of apoptosis index. TUNEL staining revealed evidence of cell apoptosis in the lung. LPS administration increased the number of apoptotic cells (red arrow) and apoptosis index. 4 weeks of exercise intervention decreased the number of apoptotic cells and apoptosis index in COPD mice. ${ }^{\#} P<0.05$ compared with the Con group. ${ }^{*} P<0.05$ compared with the COPD group (magnification, $x 200$ ).
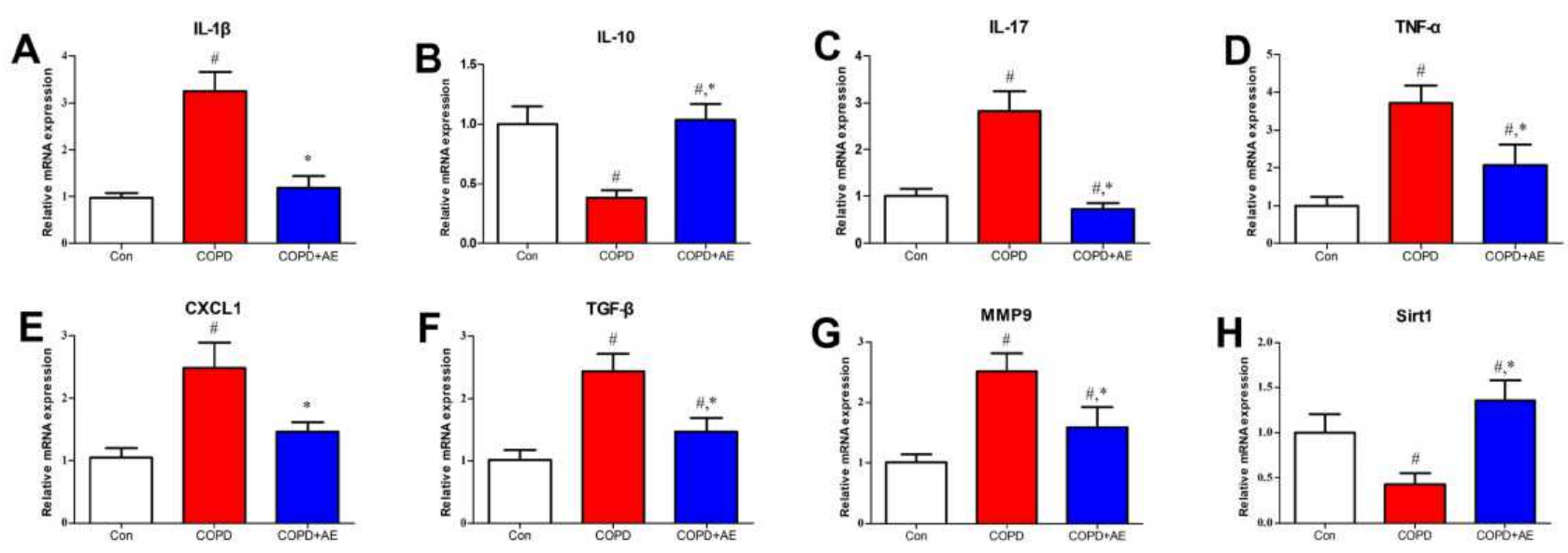

Figure 6 Detection of oxidative stress injury.

Notes: The levels of MDA (A), MPO (B), GSH (C), and SOD (D) as indicators of oxidative stress injury were detected. LPS administration increased the levels of MDA and MPO and decreased the levels of SOD and GSH. 4 weeks of exercise intervention decreased the levels of MDA and MPO and increased the levels of SOD and GSH in mice with COPD. ${ }^{\#} P<0.05$ compared with the Con group. ${ }^{*} P<0.05$ compared with the COPD group.

\section{Conclusions}

$\mathrm{AE}$ is a potential therapeutic approach for treating COPD.

$\mathrm{AE}$ improved COPD by counteracting the COPD- associated inflammatory response, oxidative stress injury, and apoptosis. In addition, AE exhibited an anti-fibrogenic effect by inhibiting the levels of the pro-fibrogenic factors 

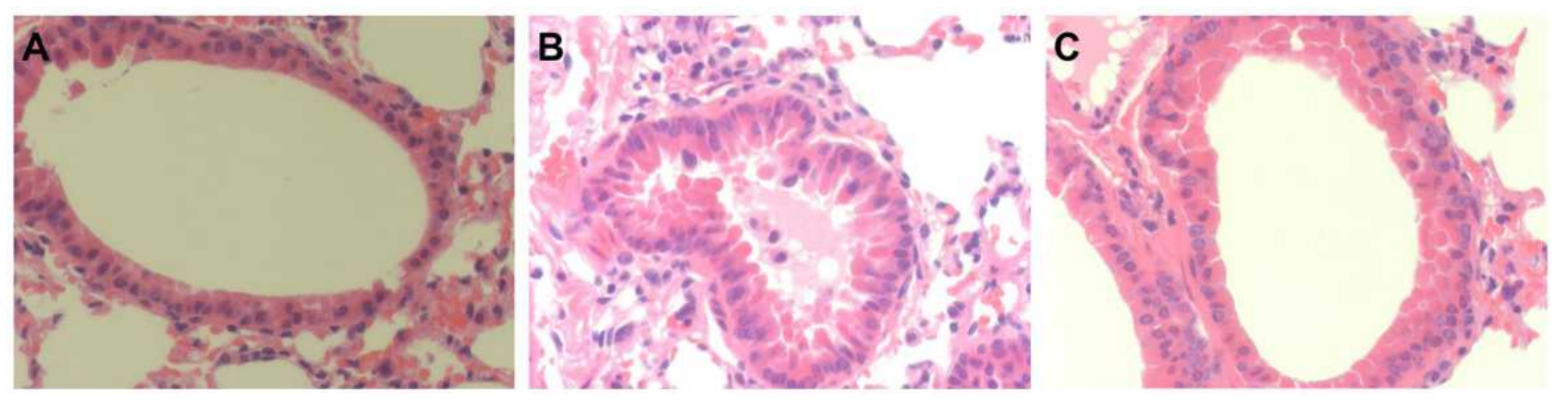

Figure 7 Detection of bronchial mucus cell hyperplasia and bronchoconstriction.

Notes: (A) Con group. (B) COPD group. (C) COPD+AE group. LPS administration led to severe bronchial mucus cell hyperplasia and bronchoconstriction, while 4 weeks of $A E$ improved bronchial mucus cell hyperplasia and bronchoconstriction (magnification, $x 400$ ).

IL-17, MMP-9, and TGF- $\beta$. Sirt1, which can be activated by regular $\mathrm{AE}$, and played a protective role in mice with COPD.

\section{Abbreviations}

$\mathrm{AE}$, aerobic exercise; $\mathrm{COPD}$, chronic obstructive pulmonary disease; BALF, bronchoalveolar lavage fluid; HE, Hematoxylin and eosin; MDA, malondialdehyde; MPO, myeloperoxidase; GSH, glutathione; SOD, superoxide dismutase; IL, interleukin; TNF, tumor necrosis factor; CXCL, C-X-C motif chemokine ligand; TGF, transforming growth factor; MMP, matrix metalloproteinase; MLI, mean linear intercept; LPS, lipopolysaccharide; PFA, paraformaldehyde; sirt, silent information regulator; dUTP, terminal deoxynucleotidyl transferase; TUNEL, nick end labeling.

\section{Data Sharing Statement}

The datasets used or analyzed during the current study are available from the corresponding author Donghui Tang on reasonable request.

\section{Acknowledgment}

This study was supported by the National Natural Science Foundation of China (NSFC) (Grant No. 71874017, 81472992), the Agricultural Science and Technology Innovation Program (ASTIP) (cxgc-ias-01), and the BNU Interdisciplinary Research Foundation for the First-Year Doctoral Candidates (Grant No. BNUXKJC1814).

\section{Disclosure}

The authors report no conflicts of interest in this work, and the manuscript has been approved by all authors for publication.

\section{References}

1. Rabe KF, Watz H. Chronic obstructive pulmonary disease. Lancet. 2017;389(10082):1931-1940. doi:10.1016/S0140-6736(17)31222-9

2. Labaki WW, Rosenberg SR. Chronic obstructive pulmonary disease. Ann Intern Med. 2020;173(3):ITC17-ITC32. doi:10.7326/AITC202008040

3. Pauwels RA, Rabe KF. Burden and clinical features of chronic obstructive pulmonary disease (COPD). Lancet. 2004;364 (9434):613-620. doi:10.1016/S0140-6736(04)16855-4

4. Barnes PJ. Immunology of asthma and chronic obstructive pulmonary disease. Nat Rev Immunol. 2008;8(3):183-192. doi:10.1038/nri2254

5. Fischer BM, Voynow JA, Ghio AJ. COPD: balancing oxidants and antioxidants. Int J Chron Obstruct Pulmon Dis. 2015;2(10):261-276. doi:10.2147/COPD.S42414

6. Sun X, Feng X, Zheng D, et al. Ergosterol attenuates cigarette smoke extract-induced COPD by modulating inflammation, oxidative stress and apoptosis in vitro and in vivo. Clin Sci. 2019;133 (13):1523-1536. doi:10.1042/CS20190331

7. Stockley RA. Neutrophils and the pathogenesis of COPD. Chest. 2002;121(5):151-155. doi:10.1378/chest.121.5_suppl.151s

8. Ong J, Faiz A, Timens W, et al. Marked TGF-beta-regulated miRNA expression changes in both COPD and control lung fibroblasts. Sci Rep. 2019;9(1):18214. doi:10.1038/s41598-019-54728-4

9. Matin S, Nemati A, Ghobadi H, et al. The effect of conjugated linoleic acid on oxidative stress and matrix metalloproteinases 2 and 9 in patients with COPD. Int $J$ Chron Obstruct Pulmon Dis. 2018;3(13):1449-1454. doi:10.2147/COPD.S155985

10. Simpson RJ, Kunz H, Agha N, et al. Exercise and the regulation of immune functions. Prog Mol Biol Transl Sci. 2015;135(355-380). doi:10.1016/bs.pmbts.2015.08.001

11. Thannickal VJ, Toews GB, White ES, et al. Mechanisms of pulmonary fibrosis. Аnnu Rev Med. 2004;55(1):395-417. doi:10.1146/ annurev.med.55.091902.103810

12. Bellaye PS, Yanagihara T, Granton E, et al. Macitentan reduces progression of TGF-beta1-induced pulmonary fibrosis and pulmonary hypertension. Eur Respir J. 2018;52(2):1701857. doi:10.1183/ 13993003.01857-2017

13. Zhang J, Wang D, Wang L, et al. Profibrotic effect of IL-17A and elevated IL-17RA in idiopathic pulmonary fibrosis and rheumatoid arthritis-associated lung disease support a direct role for IL-17A/IL17RA in human fibrotic interstitial lung disease. Am J Physiol Lung Cell Mol Physiol. 2019;316(3):L487-L497. doi:10.1152/ ajplung.00301.2018

14. Wu L, Luo Z, Zheng J, et al. IL-33 can promote the process of pulmonary fibrosis by inducing the imbalance between MMP-9 and TIMP-1. Inflammation. 2018;41(3):878-885. doi:10.1007/s10753018-0742-6 
15. Rahman I, Kinnula VL, Gorbunova V, Yao H. SIRT1 as a therapeutic target in inflammaging of the pulmonary disease. Prev Med. 2012;54:20-28. doi:10.1016/j.ypmed.2011.11.014

16. Rajendrasozhan S, Yang S-R, Kinnula VL, et al. SIRT1, an antiinflammatory and antiaging protein, is decreased in lungs of patients with chronic obstructive pulmonary disease. Am J Respir Crit Care Med. 2008;177(8):861-870. doi:10.1164/rccm.200708-1269OC

17. Yao H, Chung S, Hwang JW, et al. SIRT1 protects against emphysema via FOXO3-mediated reduction of premature senescence in mice. J Clin Invest. 2012;122(6):2032-2045. doi:10.1172/JCI60132

18. Wang X-L, Li T, Li J-H, et al. The effects of resveratrol on inflammation and oxidative stress in a rat model of chronic obstructive pulmonary disease. Molecules. 2017;22(9):1529. doi:10.3390/ molecules22091529

19. Yao H, Sundar K, Ahmad T, et al. SIRT1 protects against cigarette smoke-induced lung oxidative stress via a FOXO3-dependent mechanism. Am J Physiol Lung Cell Mol Physiol. 2014;306 (9):816-828. doi:10.1152/ajplung.00323.2013

20. Cantó C 1, Gerhart-Hines Z, Feige JN. AMPK regulates energy expenditure by modulating NAD + metabolism and SIRT1 activity. Nature. 2009;458(7241):1056-1060. doi:10.1038/nature07813

21. Stolk J, Rudolphus A, Davies P, et al. Induction of emphysema and bronchial mucus cell hyperplasia by intratracheal instillation of lipopolysaccharide in the hamster. J Pathol. 1992;167(3):349-356. doi:10.1002/path.1711670314
22. Stolk J, Rossie W, Dijkman JH. Apocynin improves the efficacy of secretory leukocyte protease inhibitor in experimental emphysema. Am J Respir Crit Care Med. 1994;150:1628-1631. doi:10.1164/ ajrccm.150.6.7952625

23. Wang X, Wang Y, Zhao X, et al. Potential effects of peroxisome proliferator-activated receptor activator on LPS-induced lung injury in rats. Pulm Pharmacol Ther. 2009;22(4):318-325. doi:10.1016/j. pupt.2009.01.004

24. Jones B, Donovan C, Liu G, et al. Animal models of COPD: what do they tell us? Respirology. 2017;22(1):21-32. doi:10.1111/resp.12908

25. Ghorani V, Boskabady MH, Khazdair MR, et al. Experimental animal models for COPD: a methodological review. Tob Induc Dis. 2017;2:15-25. doi:10.1186/s12971-017-0130-2

26. Barnes PJ. Inflammatory mechanisms in patients with chronic obstructive pulmonary disease. J Allergy Clin Immunol. 2016;138 (1):16-27. doi:10.1016/j.jaci.2016.05.011

27. Kubo H, Asai K, Kojima K, et al. Exercise ameliorates emphysema of cigarette smoke-induced COPD in mice through the exercise-irisin-Nrf2 axis. Int $J$ Chron Obstruct Pulmon Dis. 2019;14:2507-2516. doi:10.2147/COPD.S226623

\section{Publish your work in this journal}

The International Journal of COPD is an international, peer-reviewed journal of therapeutics and pharmacology focusing on concise rapid reporting of clinical studies and reviews in COPD. Special focus is given to the pathophysiological processes underlying the disease, intervention programs, patient focused education, and self management protocols. This journal is indexed on PubMed Central, MedLine and CAS. The manuscript management system is completely online and includes a very quick and fair peer-review system, which is all easy to use. Visit http://www.dovepress.com/testimonials.php to read real quotes from published authors. 